\title{
Efficacy and Safety of a Phytopharmaceutical Drug Derived from Cocculus hirsutus in Adults with Moderate COVID-19: a Phase 2, Open-label, Multicenter, Randomized Controlled Trial
}

\author{
Sadhna Joglekar (D) - Shivakumar S. Iyer - Rohit Parate • \\ Akash A. Khobragade - Rakesh Patil - Shashi Bhushan • \\ Dnyanshwar M. Halnor - Girish C. Rajadhyaksha · Kartikeya Parmar • \\ Shilpi Dhawan · Suyog Mehta · Shashank R. Joshi
}

Received: November 10, 2021 / Accepted: February 1, 2022 / Published online: February 18, 2022

(C) The Author(s) 2022

\begin{abstract}
Introduction: There is an urgent need for an effective, oral therapy for COVID-19. Purified aqueous extract of Cocculus hirsutus (AQCH) has shown robust antiviral activity in in vitro studies. We aimed to evaluate the efficacy and safety of AQCH plus standard of care in hospitalized patients with moderate COVID-19.
\end{abstract}

Supplementary Information The online version contains supplementary material available at https:// doi.org/10.1007/s40121-022-00604-0.

S. Joglekar $(\bowtie) \cdot$ S. Dhawan · S. Mehta

Sun Pharmaceutical Industries Limited, SUN

HOUSE, CTS No. 201 B/1, Western Express

Highway, Goregaon (East), Mumbai, Maharashtra 400063, India

e-mail: sadhna.joglekar@sunpharma.com

\section{S. S. Iyer}

Department of Critical Care Medicine, Bharati

Vidyapeeth (Deemed to be University) Medical

College, Pune, Maharashtra, India

\section{R. Parate}

Department of Medicine, Chirayu Hospital, Bhopal, Madhya Pradesh, India

\section{A. A. Khobragade}

St George's Hospital, Mumbai, Maharashtra, India

R. Patil

Department of General Medicine, Apex Hospitals,

Mumbai, Maharashtra, India
Methods: In an open-label, multicenter, randomized controlled trial conducted in India, eligible patients (aged 18-75 years) were randomized (1:1) to receive AQCH $400 \mathrm{mg}$ orally three times a day plus standard of care $(\mathrm{AQCH}$ group) or standard of care alone (control group) for 10 days. Primary endpoint was the proportion of patients showing clinical improvement by day 14 . Time to clinical improvement, time to viral clearance, and duration of hospitalization were secondary endpoints.

\section{S. Bhushan}

Department of Pulmonology, Bangalore Medical

College and Victoria Hospital, Bengaluru,

Karnataka, India

D. M. Halnor

Department of Clinical Research, Vijay Vallabh

Hospital, Virar, Maharashtra, India

G. C. Rajadhyaksha

Department of Medicine, Topiwala National

Medical College and B.Y.L. Nair Charitable Hospital, Mumbai, Maharashtra, India

\section{K. Parmar}

Department of Medicine, B.J. Medical College and Civil Hospital, Ahmedabad, Gujarat, India

\section{S. R. Joshi}

Departments of Endocrinology, Joshi Clinic and Lilavati Hospital, Mumbai, Maharashtra, India 
Results: A total of 210 patients were randomized. By day 14 most patients in both groups showed clinical improvement [difference -0.01 (95\% CI -0.07 to 0.05 ); $p=1.0]$. Median time to clinical improvement was 8 days (IQR 8-11) in the AQCH group versus 11 days (IQR 8-11) in the control group [HR 1.27 (95\% CI 0.95-1.71); $p=0.032]$. Time to viral clearance and duration of hospitalization were also significantly shorter in the AQCH group $(p=0.0002$ and $p=0.016$, respectively). AQCH was well tolerated, with no safety concerns identified.

Conclusions: AQCH significantly reduced time to clinical improvement, time to viral clearance, and duration of hospitalization. In a pandemic, this has significant potential to decrease healthcare resource utilization and increase hospital bed availability. Further investigation of the therapeutic potential of $A Q C H$ in patients with COVID-19 is warranted.

Trial Registration: Clinical Trials Registry India (CTRI/2020/05/025397).

Keywords: Antiviral; Cocculus hirsutus; COVID19; Phytopharmaceutical; Randomized controlled trial

\section{Key Summary Points}

\section{Why carry out this study?}

Purified aqueous extract of Cocculus hirsutus (AQCH) has shown robust antiviral activity in in vitro studies, and there is an urgent need for an effective, oral therapy for COVID-19.

This is the first randomized, controlled trial assessing the efficacy and safety of oral treatment with AQCH in hospitalized patients with moderate COVID-19.

\section{What was learned from the study?}

Patients who received AQCH had significantly faster clinical improvement and viral clearance, as well as significantly shorter duration of hospitalization, versus patients who received standard of care alone.
These data provide support for the continued investigation of $\mathrm{AQCH}$ as a treatment for patients with COVID-19, with the potential to speed patient recovery and decrease healthcare burden.

This study also highlights key considerations for the selection of clinical trial endpoints in a relatively new and poorly understood disease and the importance of considering study findings holistically in the context of current understanding.

\section{INTRODUCTION}

Coronavirus disease 2019 (COVID-19), caused by severe acute respiratory syndrome coronavirus 2 (SARS-CoV-2), has overwhelmed healthcare systems around the world. Although most people with COVID-19 are asymptomatic or experience only mild disease, a significant proportion of patients require hospitalization and may become critically ill $[1,2]$. As of October 2021, more than 240 million cases and over 4.9 million deaths have been reported worldwide [3]. In India alone, more than 34 million COVID-19 cases have been reported, although actual numbers may be much higher owing to limitations in testing and the spread of the disease to rural areas with limited access to healthcare [3-5].

In response to the pandemic, the scientific and research community has rapidly directed efforts into developing or repurposing drugs for the treatment of COVID-19. The antiviral drugs remdesivir and favipiravir have received emergency use authorization in several countries based on limited data [6, 7]; however, studies of these therapies in patients with COVID-19 have shown inconsistent results [8-16]. In addition, vaccination programs in many resource-limited countries may take years to have a significant impact at population levels. As countries contend with multiple waves of infections and the emergence of new SARS-CoV-2 variants [4, 17], there remains an urgent need for an oral 
therapy that can both speed patient recovery and limit community transmission.

Purified aqueous extract of Cocculus hirsutus $(\mathrm{AQCH})$ is a phytopharmaceutical drug with potential as a novel treatment for COVID-19. Cocculus hirsutus is a tropical plant found in parts of Africa and Asia, which has been widely used in traditional medicine owing to its analgesic and anti-inflammatory properties [18]. $\mathrm{AQCH}$ is in clinical development as an antiviral drug and has undergone comprehensive preclinical studies, including acute and repeated dose toxicity studies of oral AQCH in rodent and non-rodent species, as well as a phase 1 clinical trial (manuscripts submitted). AQCH demonstrated robust antiviral activity in in vitro studies and in an animal model of dengue [19]. Five chemical compounds have been characterized in AQCH, of which only sinococuline has potent pan-anti-dengue activity [19]. In vitro mechanism-of-action studies indicate that AQCH interferes in the early stages of viral replication, and has inhibitory activity against SARS-CoV-2 with a half-maximal inhibitory concentration $\left(\mathrm{IC}_{50}\right)$ of $6.8 \mu \mathrm{g} / \mathrm{mL}$ (manuscript in preparation). In a randomized, double-blind, placebo-controlled, multiple ascending dose, safety, and tolerability study in healthy volunteers, AQCH given orally in doses of 100-800 $\mathrm{mg}$ three times a day for 10 days was well tolerated. Incidences of adverse events were similar in individuals who received AQCH or placebo, with no serious adverse events, and all events were resolved.

Here, we report the results of a phase 2, randomized controlled trial in which we aimed to evaluate the efficacy and safety of AQCH plus standard of care versus standard of care alone in hospitalized patients with moderate COVID-19.

\section{METHODS}

\section{Study Design}

This was a phase 2, open-label, parallel-group, randomized controlled trial conducted at eight hospitals in four states (Gujarat, Karnataka, Madhya Pradesh, Maharashtra), in India (Clinical Trials Registry - India: CTRI/2020/05/
025397). Approval was obtained from the national regulatory agency and institutional ethics committee at each hospital before study initiation. The study was conducted in accordance with the ethical principles of the Declaration of Helsinki, Good Clinical Practice guidelines issued by the Central Drugs Standard Control Organization, and the National Guidelines for Ethics Committees Reviewing Biomedical and Health Research During COVID-19 Pandemic issued by the Indian Council of Medical Research (April 2020). All participants provided written informed consent.

\section{Patients}

Eligible patients were men and non-pregnant, non-lactating women aged $18-75$ years with a body temperature over $37.3^{\circ} \mathrm{C}$, cough and/or shortness of breath, and moderate COVID-19 infection with either (1) a ratio of partial pressure arterial oxygen to fraction of inspired oxygen $\left(\mathrm{PaO}_{2} / \mathrm{FiO}_{2}\right)$ of $200-300$, or (2) respiratory rate above 24 breaths per min with oxygen saturation of $93 \%$ or lower on room air. An RTPCR-confirmed diagnosis of COVID-19 was required for all participants. Patients were excluded from the study if they had any concurrent medical condition, or uncontrolled, clinically significant systemic disease that, in the opinion of the investigator, precluded the patient's participation in the study or would interfere with interpretation of study results. Other exclusion criteria were persistent vomiting, active hepatitis, tuberculosis, and definite bacterial or fungal infections. If a patient's condition worsened significantly $\left(\mathrm{PaO}_{2} /\right.$ $\mathrm{FiO}_{2}<150$ or shock requiring a vasopressor to maintain a mean arterial pressure of $\geq 65 \mathrm{~mm} \mathrm{Hg}$ ), they were withdrawn from the study.

\section{Study Procedures}

Patients were assessed for study eligibility and baseline characteristics during a screening period of $24-48 \mathrm{~h}$. Each patient was assigned a unique study randomization number 
(ascending consecutive order within each site). They were then randomized $(1: 1)$ to receive either tablets of purified AQCH $400 \mathrm{mg}$ orally three times a day ( $\geq 30$ min before a meal) plus standard of care or standard of care alone for 10 days (treatment period). This dose was selected based on phase 1 pharmacokinetic data available at the time of protocol development, which indicated safety and tolerability of AQCH in heathy volunteers and a linear increase in peak plasma concentration $\left(C_{\max }\right)$ and area under the concentration-time curve (AUC), with ascending doses of up to $400 \mathrm{mg}$ three times a day. The preparation, standardization, and stability of AQCH tablets have been described previously [19].

Randomization was based on a computergenerated schedule prepared prior to study initiation (SAS software, version 9.4.). Standard of care was provided as per institutional practice. Patients were hospitalized throughout the treatment period and monitored for clinical symptoms, body temperature, vital signs, concomitant medications, and adverse events. Scheduled assessments included RT-PCR for SARS-CoV-2 (days 3, 7, and 10), arterial blood gases (days 1, 3, 5, 7, and 10), 12-lead electrocardiogram (days 5 and 10), hematology (days 3,5 , and 7), biochemistry (day 7), exploratory prognostic markers (C-reactive protein, D-dimer, ferritin, and lactate dehydrogenase; days 3 and 7 if feasible), and chest X-ray/CT scan (day 7). Arterial blood gases, electrocardiogram, hematology, biochemistry, and chest $\mathrm{X}$-ray/CT scan were assessed on day 14 (or day of discharge) and at follow-up. Hospital discharge criteria were resolution of symptoms and radiological improvement with documented viral clearance by RT-PCR in two samples at least $24 \mathrm{~h}$ apart. All patients were assessed on day 14 (or day of discharge) and completed a follow-up visit 28 days after randomization ( \pm 3 days). Details of data collection can be found in the Supplementary Methods.

\section{Study Outcomes}

The primary outcome was the proportion of patients showing clinical improvement by day
14 , defined as meeting hospital discharge criteria or having a two-point improvement in disease severity from time of enrollment on a seven-point ordinal scale $(1=$ not hospitalized with resumption of normal activities; $2=$ not hospitalized but unable to resume normal activities; $3=$ hospitalized, not requiring supplemental oxygen; $4=$ hospitalized, requiring supplemental oxygen; $5=$ hospitalized, requiring nasal high-flow oxygen therapy or non-invasive mechanical ventilation, or both; $6=$ hospitalized, requiring extracorporeal membrane oxygenation or invasive mechanical ventilation, or both; $7=$ death).

Secondary outcomes were: proportions of patients showing clinical improvement by days 7 and 28; time to clinical improvement; time to normalization of fever without use of antipyretics in the past $24 \mathrm{~h}$; time to alleviation of cough; time to viral clearance (first negative respiratory tract SARS-CoV-2 RT-PCR test); duration of supplemental oxygen therapy; proportion of patients showing deterioration of clinical condition ( $\geq 1$-point worsening on the ordinal scale); duration of hospitalization (time to meeting hospital discharge criteria); and number of deaths. Safety outcomes included treatment-emergent adverse events and serious adverse events. Severity of adverse events was reported using Common Terminology Criteria for Adverse Events, version 5.0.

\section{Statistical Analysis}

Based on assumption of clinical improvement in $90 \%$ and $75 \%$ of patients in the test and control groups, respectively, and considering $80 \%$ power, a sample size of 105 patients per group was chosen. Patient demographics and baseline characteristics were summarized for all randomized patients (intention-to-treat population) using descriptive statistics. The modified intention-to-treat population included all randomized patients who met all inclusion/exclusion criteria, received at least one dose of study medication, and had at least one post-baseline evaluation. Patients who completed the study period without any major protocol deviations 


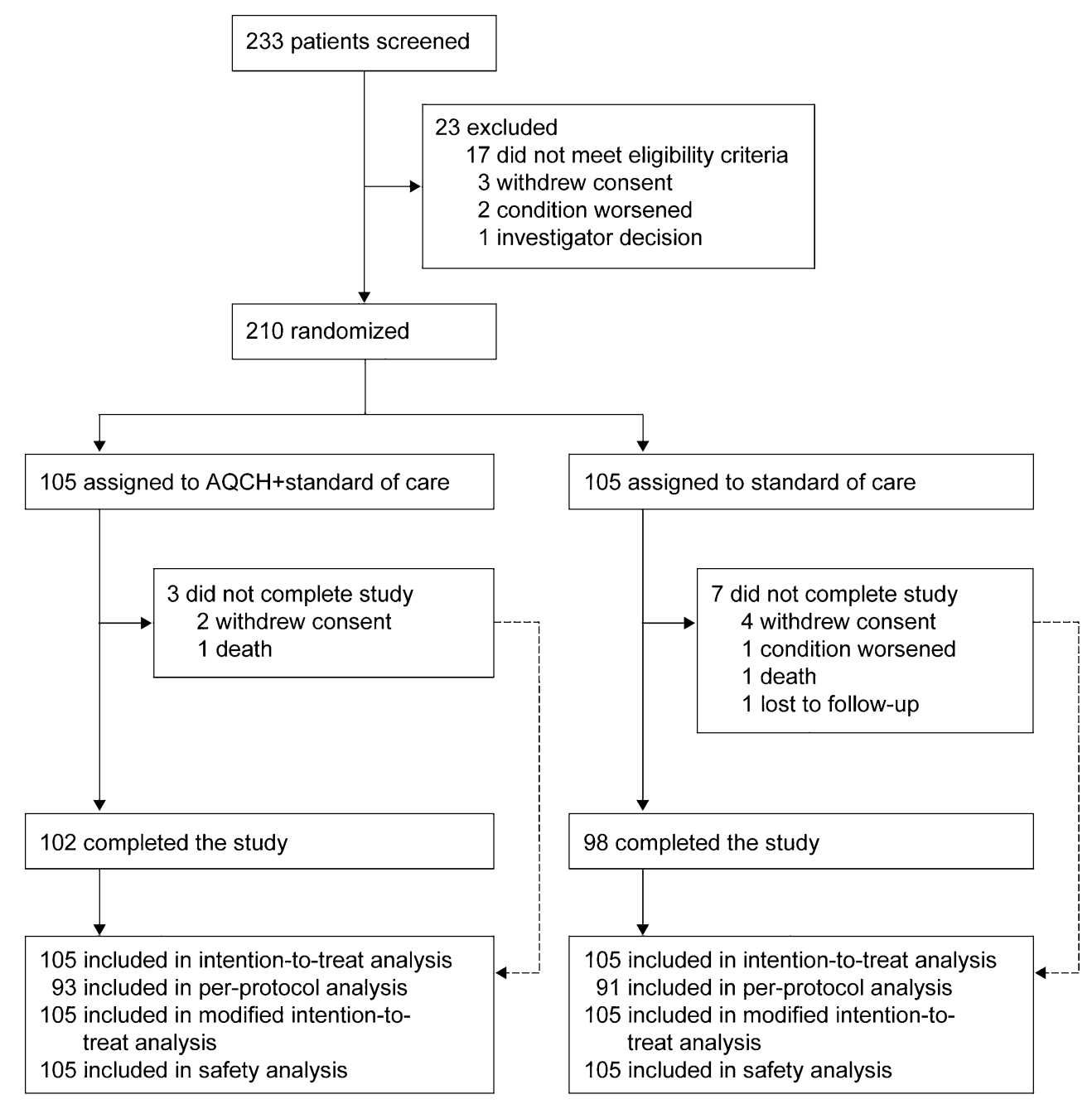

Fig. 1 Trial profile. $A Q C H$ aqueous extract of Cocculus hirsutus

were included in efficacy analyses (per-protocol population).

The primary efficacy endpoint was compared between groups using Fisher's exact test. Difference in clinical improvement response rate was summarized using point estimate and 95\% confidence interval (CI). These methods were also used for analyses of clinical improvement at days 7 and 28. Time-to-event secondary endpoints were compared between groups using a log-rank test with time to event as the dependent variable and treatment group as the test variable. Hazard ratios (HRs) were estimated using a Cox regression model with treatment as the only covariant. The Kaplan-Meier method was used to estimate median time to event, with generation of Kaplan-Meier curves. Ad hoc analyses of the proportions of patients in each group who met time-to-event endpoints on each day were performed using the chi-squared test (Fisher's exact test in case of extremes). Owing to difficulties in SARS-CoV-2 RT-PCR testing during the pandemic, tests performed within 1 day of the protocol-defined day were considered minor protocol deviations and did not preclude patients from inclusion in the perprotocol population. Analyses of viral clearance were performed in both the per-protocol population and in a subset of patients for whom RTPCR tests were conducted on the protocol-defined days. Duration of supplemental oxygen therapy and duration of hospitalization were 
Table 1 Baseline patient characteristics

AQCH + standard of care $(n=105) \quad$ Standard of care $(n=105)$

Sex

Male

Female

Age, years, mean (SD)

$$
<45
$$

$45-60$

$>60$

Weight, kg, mean (SD)

BMI, $\mathrm{kg} / \mathrm{m}^{2}$, mean (SD)

Comorbid conditions

Any

Diabetes

Hypertension

Diabetes + hypertension

Cerebral infarction

Palpitations

Clinical status

3

4

5

Body temperature, ${ }^{\circ} \mathrm{C}$, mean $(\mathrm{SD})$

Fever $>37.3^{\circ} \mathrm{C}$

With cough

With shortness of breath

With cough and shortness of breath

$\mathrm{PaO}_{2} / \mathrm{FiO}_{2}$ ratio, mean (SD)

$$
<200
$$

200-300

$>300$

$\mathrm{SaO}_{2}$ or $\mathrm{SpO}_{2}, \%$, mean (SD)

$$
<90
$$

90-93
$76(72)$

29 (28)

44.7 (12.7)

$54(51)$

39 (37)

12 (11)

$68.9(12.0)$

26.5 (5.7)

14 (13)

4 (4)

$5(5)$

3 (3)

1 (1)

1 (1)

28 (27)

76 (72)

1 (1)

$38.0(0.5)$

29 (28)

21 (20)

$55(52)$

$281.2(82.8)$

1 (1)

83 (79)

21 (20)

$90.6(1.8)$

23 (22)

$82(78)$
38 (36)

64 (61)

$74(70)$

31 (30)

47.6 (13.3)

45 (43)

38 (36)

22 (21)

68.6 (11.3)

26.7 (6.6)

21 (20)

$10(10)$

$6(6)$

$5(5)$

0

0

3 (3)

$38.0(0.5)$

$33(31)$

24 (23)

48 (46)

$276.1(90.0)$

3 (3)

88 (84)

14 (13)

90.5 (2.2)

28 (27)

77 (73) 
Table 1 continued

AQCH + standard of care $(n=105)$

Respiratory rate, breaths per min, mean (SD) $26.6(1.4) \quad 26.6(1.4)$

Standard of care $(n=105)$

Respiratory rate, breaths per min, mean (SD) $26.6(1.4) \quad 26.6(1.4)$

Data are $n$ (\%) unless otherwise specified. Clinical status was assessed using a 7-point ordinal scale for disease severity ( $1=$ not hospitalized with resumption of normal activities; $2=$ not hospitalized but unable to resume normal activities; $3=$ hospitalized, not requiring supplemental oxygen; $4=$ hospitalized, requiring supplemental oxygen; $5=$ hospitalized, requiring nasal high-flow oxygen therapy or non-invasive mechanical ventilation, or both; $6=$ hospitalized, requiring extracorporeal membrane oxygenation or invasive mechanical ventilation, or both; $7=$ death)

AQCH aqueous extract of Cocculus hirsutus, $B M I$ body mass index, $\mathrm{PaO}_{2} / \mathrm{FiO}_{2}$ partial pressure arterial oxygen to fraction of inspired oxygen, $\mathrm{SaO}_{2}$ arterial oxygen saturation, $\mathrm{SpO}_{2}$ arterial oxygen saturation measured by pulse oximetry

compared between treatment groups using a $t$ test or Wilcoxon rank sum test depending on normality. All analyses were performed using SAS software, version 9.4.

All randomized patients who received at least one dose of study medication were included in safety analyses (safety population). An independent data safety monitoring board (DSMB) evaluated data from two interim safety analyses, after enrollment of 40 patients and 102 patients. Based on the results of the interim analyses, the DSMB assessed whether to modify, terminate, or continue the study.

\section{RESULTS}

\section{Patients}

Between June and July 2020, a total of 233 patients were screened, of whom 105 were randomly assigned to receive AQCH plus standard of care (AQCH group) and 105 were assigned to receive standard of care alone (control group; Fig. 1). Final follow-up was in August 2020. A total of 200 patients completed the study, of whom 184 had no major protocol deviations and were included in the per-protocol population: 93 in the AQCH group and 91 in the control group. All major protocol deviations (AQCH group $n=9$, control group $n=7$ ) were related to RT-PCR testing (test performed $>1$ day from protocol-defined day or patient discharged despite positive test).

Baseline demographics and clinical characteristics were well balanced between the treatment groups (Table 1). Overall mean age was 46.2 years (SD 13.0) and mean body mass index was $26.6 \mathrm{~kg} / \mathrm{m}^{2}$ (SD 6.2). Of the 210 patients, 150 (71\%) were men. Overall, 35 patients (17\%) had comorbid conditions. Standard-of-care treatments were prescribed based on institutional practice and at the investigator's discretion (Table 2). Corticosteroids were used in only 21 patients (10\%) and 147 patients (70\%) received oxygen.

\section{Efficacy}

The primary endpoint of proportion of patients with clinical improvement by day 14 was $97 \%$ (90 of 93 patients) in the AQCH group and 98\% (89 of 91) in the control group (Table 3 ). The difference between the two groups was not statistically significant. No significant difference was observed for the secondary endpoints of proportions of patients with clinical improvement by days 7 and 28 (Table 3). Median time to clinical improvement was significantly shorter in the AQCH group [8 days (IQR 8-11)] than in the control group [11 days (IQR 8-11); HR 1.27 (95\% CI 0.95-1.71); $p=0.032$; Table 3; Fig. 2i]. The proportion of patients with clinical improvement was significantly greater in the $\mathrm{AQCH}$ group versus the control group on each day from day 8 to day 13 (Fig. 2ii). Results were similar when assessing patients with at least a one-point improvement in disease severity, with a significant difference between the two treatment groups observed from day 6 (Fig. 3i). The proportion of patients with a baseline score of 4 (AQCH group $n=64$, control group $n=54$ ) 
Table 2 Details of standard of care

\begin{tabular}{|c|c|c|}
\hline & $\begin{array}{l}\text { AQCH + standard of } \\
\text { care }(n=105)\end{array}$ & $\begin{array}{l}\text { Standard of care } \\
(n=105)\end{array}$ \\
\hline All other therapeutic products & $76(72)$ & $71(68)$ \\
\hline Oxygen & $76(72)$ & $71(68)$ \\
\hline Anti-emetics and antinauseants & 0 & $1(1 \%)$ \\
\hline Diphenhydramine & 0 & $1(1 \%)$ \\
\hline Antimalarials & $62(59)$ & $62(59)$ \\
\hline Hydroxychloroquine & $31(30)$ & $31(30)$ \\
\hline Hydroxychloroquine sulphate & $31(30)$ & $31(30)$ \\
\hline Antinematodal agents & $7(7)$ & $5(5)$ \\
\hline Ivermectin & $7(7)$ & $5(5)$ \\
\hline Antithrombotic agents & $22(21)$ & $24(23)$ \\
\hline Enoxaparin & $15(14)$ & $16(15)$ \\
\hline Low-molecular-weight heparin & $6(6)$ & $6(6)$ \\
\hline Heparin & $2(2)$ & $3(3)$ \\
\hline Ascorbic acid (vitamin C) & $89(85)$ & $88(84)$ \\
\hline Ascorbic acid & $89(85)$ & $88(84)$ \\
\hline Beta-lactam antibacterials, penicillins & 0 & $1(1)$ \\
\hline Phenoxymethylpenicillin & 0 & $1(1)$ \\
\hline Calcium & $16(15)$ & $17(16)$ \\
\hline Calcium carbonate; colecalciferol & $16(15)$ & $17(16)$ \\
\hline Corticosteroids for systemic use, plain & $9(9)$ & $12(11)$ \\
\hline Methylprednisolone & $7(7)$ & $8(8)$ \\
\hline Dexamethasone & $2(2)$ & $5(5)$ \\
\hline Cough suppressants and expectorants, combinations & 0 & $1(1)$ \\
\hline $\begin{array}{l}\text { Ammonium chloride; chlorphenamine maleate; dextromethorphan } \\
\text { hydrobromide; guaifenesin }\end{array}$ & 0 & $1(1)$ \\
\hline Cough suppressants, excluding combinations with expectorants & $1(1)$ & $3(3)$ \\
\hline Dextromethorphan hydrobromide & $1(1)$ & 0 \\
\hline Chlorphenamine; dextromethorphan; phenylephrine & 0 & $3(3)$ \\
\hline Direct acting antivirals & $20(19)$ & $20(19)$ \\
\hline Oseltamivir & $16(15)$ & $16(15)$ \\
\hline Oseltamivir phosphate & $4(4)$ & $5(5)$ \\
\hline Drugs for peptic ulcer and gastro-esophageal reflux disease & $6(6)$ & $6(6)$ \\
\hline
\end{tabular}


Table 2 continued

\begin{tabular}{|c|c|c|}
\hline & $\begin{array}{l}\text { AQCH + standard of } \\
\text { care }(n=105)\end{array}$ & $\begin{array}{l}\text { Standard of care } \\
(n=105)\end{array}$ \\
\hline Pantoprazole & $6(6)$ & $6(6)$ \\
\hline Pantoprazole sodium sesquihydrate & $1(1)$ & 0 \\
\hline Expectorants, excluding combinations with cough suppressants & $26(25)$ & $26(25)$ \\
\hline Acetylcysteine & $15(14)$ & $15(14)$ \\
\hline Bromhexine hydrochloride; guaifenesin; salbutamol sulphate & $7(7)$ & $11(10)$ \\
\hline Ambroxol; guaifenesin; levosalbutamol & $4(4)$ & 0 \\
\hline Intravenous solutions & $4(4)$ & $5(5)$ \\
\hline Sodium chloride & $3(3)$ & $5(5)$ \\
\hline Glucose; sodium chloride & $1(1)$ & 0 \\
\hline Macrolides, lincosamides, and streptogramins & $59(56)$ & $64(61)$ \\
\hline Azithromycin & $59(56)$ & $64(61)$ \\
\hline Multivitamins, combinations & $15(14)$ & $14(13)$ \\
\hline Minerals; vitamins & $15(14)$ & $14(13)$ \\
\hline Multivitamins, plain & $25(24)$ & $23(22)$ \\
\hline Vitamins & $25(24)$ & $23(22)$ \\
\hline Other alimentary tract and metabolism products & $2(2)$ & $4(4)$ \\
\hline Zinc acetate & $2(2)$ & $4(4)$ \\
\hline Other analgesics and antipyretics & $103(98)$ & $103(98)$ \\
\hline Paracetamol & $103(98)$ & $103(98)$ \\
\hline Ibuprofen; paracetamol & 0 & $2(2)$ \\
\hline Other beta-lactam antibacterials & $25(24)$ & $22(21)$ \\
\hline Cefixime & $16(15)$ & $12(11)$ \\
\hline Meropenem & $9(9)$ & $8(8)$ \\
\hline Cefotaxime sodium & $1(1)$ & 0 \\
\hline Ceftriaxone sodium & 0 & $3(3)$ \\
\hline Other mineral supplements & $22(21)$ & $20(19)$ \\
\hline Zinc & $22(21)$ & $20(19)$ \\
\hline Other vitamin products, combinations & $22(21)$ & $22(21)$ \\
\hline $\begin{array}{l}\text { Ascorbic acid; biotin; chromium; colecalciferol; copper; folic acid; iodine; } \\
\text { magnesium; manganese; nicotinamide; pantothenic acid; pyridoxine } \\
\text { hydrochloride; retinol; riboflavin; selenium; vitamin B1; vitamin B12 }\end{array}$ & $15(14)$ & $13(12)$ \\
\hline Vitamin B & $5(5)$ & $6(6)$ \\
\hline
\end{tabular}


Table 2 continued

\begin{tabular}{lll}
\hline & $\begin{array}{l}\text { AQCH + standard of } \\
\text { care }(\boldsymbol{n}=\mathbf{1 0 5})\end{array}$ & $\begin{array}{l}\text { Standard of care } \\
(\boldsymbol{n}=\mathbf{1 0 5})\end{array}$ \\
\hline $\begin{array}{l}\text { Ascorbic acid; calcium pantothenate; folic acid; nicotinamide; pyridoxine } \\
\text { hydrochloride; riboflavin; thiamine hydrochloride; vitamin B12; zinc } \\
\text { sulphate monohydrate }\end{array}$ & $3(2)$ & \\
Tetracyclines & $13(12)$ & $12(11)$ \\
Doxycycline & $13(12)$ & $12(11)$ \\
Throat preparations & $6(6)$ & 0 \\
Povidone-iodine & $6(6)$ & 0 \\
Vitamin B-complex, including combinations & $2(2)$ & $1(1)$ \\
Ascorbic acid; vitamin B & $2(2)$ & $1(1)$ \\
\hline
\end{tabular}

Data are $n$ (\%) by Anatomical Therapeutic Chemical Class 3 and preferred term $A Q C H$ aqueous extract of Cocculus hirsutus

who had at least a one-point clinical improvement was numerically higher in the AQCH group than in the control group throughout the first week of treatment (Fig. 3ii).

Median time to normalization of fever without the use of antipyretics was 5 days (IQR $4-8$ ) in the AQCH group versus 6 days (IQR 5-9) in the control group [HR $1.30 \quad(95 \%$ CI 0.97-1.74); $p=0.037$; Table 3; Supplementary Fig. S1]. In patients with no protocol deviations in RT-PCR testing (AQCH group $n=83$, control group $n=84$ ), median time to viral clearance was significantly shorter in the AQCH group [7 days (IQR 7-7)] versus the control group [10 days (IQR 7-10); HR $1.44 \quad(95 \%$ CI 1.05-1.96); $p=0.0002 ;$ Table 3; Fig. 4]. The proportion of patients with a negative SARSCoV-2 RT-PCR test was significantly greater in the AQCH group (77\%) than in the control group (49\%) on day 7 ( $p=0.0002$; Table 3$)$. This trend was already apparent by day 3 ; by day 10 , viral clearance rates were similar between treatment groups. Median time to viral clearance was also significantly shorter in the AQCH group versus the control group when the full per-protocol population was considered $(p=0.010 ;$ Supplementary Fig. S2).
Median duration of hospitalization was 11 days (IQR 9-12) in the AQCH group and 12 days (IQR 10-13) in the control group $(p=0.016$; Table 3$)$. From day 8 , the proportion of patients meeting hospital discharge criteria was consistently numerically greater in the $\mathrm{AQCH}$ group versus the control group, reaching statistical significance at days 11 and 12 (Fig. 5). Deterioration of clinical condition was reported for 17 of 105 patients (16\%) in the AQCH group and 20 of 105 patients (19\%) in the control group; the difference between the groups was not statistically significant (Table 3). No significant between-group differences were observed in time to alleviation of cough, duration of supplemental oxygen therapy, and number of deaths (Supplementary Table S1).

Similar trends were observed for all efficacy endpoints when assessed in the modified intention-to-treat population, which included patients with major protocol deviations (Supplementary Table S2).

\section{Safety}

Adverse events were reported in 12 (11\%) of 105 patients in the AQCH group and seven (7\%) of 
Table 3 Outcomes in the per-protocol population

\begin{tabular}{|c|c|c|c|c|}
\hline & $\begin{array}{l}\text { AQCH + standard of } \\
\text { care }(n=93)\end{array}$ & $\begin{array}{l}\text { Standard of care } \\
(n=91)\end{array}$ & $\begin{array}{l}\text { Difference/HR } \\
(95 \% \mathrm{CI})\end{array}$ & $p$ value \\
\hline \multicolumn{5}{|l|}{ Primary endpoint } \\
\hline $\begin{array}{l}\text { Patients with clinical improvement by day } \\
14\end{array}$ & $90(97)$ & $89(98)$ & $\begin{array}{l}-0.01(-0.07 \\
\text { to } 0.05)\end{array}$ & 1.0 \\
\hline \multicolumn{5}{|l|}{ Secondary endpoints } \\
\hline Patients with clinical improvement by day 7 & $11(12)$ & $8(9)$ & $\begin{array}{l}0.03(-0.06 \text { to } \\
0.12)\end{array}$ & 0.50 \\
\hline $\begin{array}{l}\text { Patients with clinical improvement by day } \\
28\end{array}$ & $93(100)$ & $91(100)$ & $\begin{array}{l}0(-0.04 \text { to } \\
0.04)\end{array}$ & NC \\
\hline Time to clinical improvement, days & $8(8-11)$ & $11(8-11)$ & $\begin{array}{l}\text { HR } 1.27 \\
\quad(0.95-1.71)\end{array}$ & 0.032 \\
\hline Time to normalization of fever, days & $5(4-8)$ & $6(5-9)$ & $\begin{array}{l}\text { HR } 1.30 \\
\qquad(0.97-1.74)\end{array}$ & 0.037 \\
\hline Time to viral clearance, days ${ }^{\mathrm{a}}$ & $7(7-7)$ & $10(7-10)$ & $\begin{array}{l}\text { HR } 1.44 \\
\quad(1.05-1.96)\end{array}$ & 0.0002 \\
\hline \multicolumn{5}{|l|}{ Viral clearance rates ${ }^{\mathrm{a}}$} \\
\hline Day 3 & $14(17)$ & $10(12)$ & $\begin{array}{l}0.05(-0.06 \text { to } \\
0.16)\end{array}$ & 0.36 \\
\hline Day 7 & $64(77)$ & $41(49)$ & $0.28(0.14-0.41)$ & 0.0002 \\
\hline Day 10 & $83(100)$ & $82(98)$ & $\begin{array}{l}0.02(-0.02 \text { to } \\
0.08)\end{array}$ & 0.50 \\
\hline $\begin{array}{l}\text { Proportion of patients showing } \\
\text { deterioration of clinical condition }{ }^{\mathrm{b}}\end{array}$ & $17(16)$ & $20(19)$ & $\begin{array}{l}-0.03(-0.13 \\
\text { to } 0.08)\end{array}$ & 0.59 \\
\hline Duration of hospitalization, days & $11(9-12)$ & $12(10-13)$ & - & 0.016 \\
\hline
\end{tabular}

Data are $n$ (\%) or median (IQR). Clinical improvement was defined as meeting hospital discharge criteria or a 2-point improvement from time of enrollment in disease severity rating on a 7-point ordinal scale. Viral clearance was defined as negative SARS-CoV-2 RT-PCR test. Deterioration of clinical condition was defined as $\geq 1$-point worsening on the ordinal scale. Duration of hospitalization was calculated as time to meeting hospital discharge criteria. Tests for statistical comparisons: chi-squared test/Fisher's exact test for endpoints on specific days; log-rank test for time-to-event endpoints; Wilcoxon rank sum test for duration of hospitalization

$A Q C H$ aqueous extract of Cocculus hirsutus, $H R$ hazard ratio, $N C$ not calculated, SARS-CoV-2 severe acute respiratory syndrome coronavirus 2

${ }^{a}$ Analyses of viral clearance were conducted in patients with no minor protocol deviations in RT-PCR testing (patients in whom RT-PCR was conducted on the protocol-scheduled days; AQCH + standard of care $n=83$, standard of care $n=84)$

${ }^{\mathrm{b}}$ Analyses of clinical deterioration were conducted in the modified intention-to-treat population $(\mathrm{AQCH}+$ standard of care $n=105$, standard of care $n=105$ ) 
i)

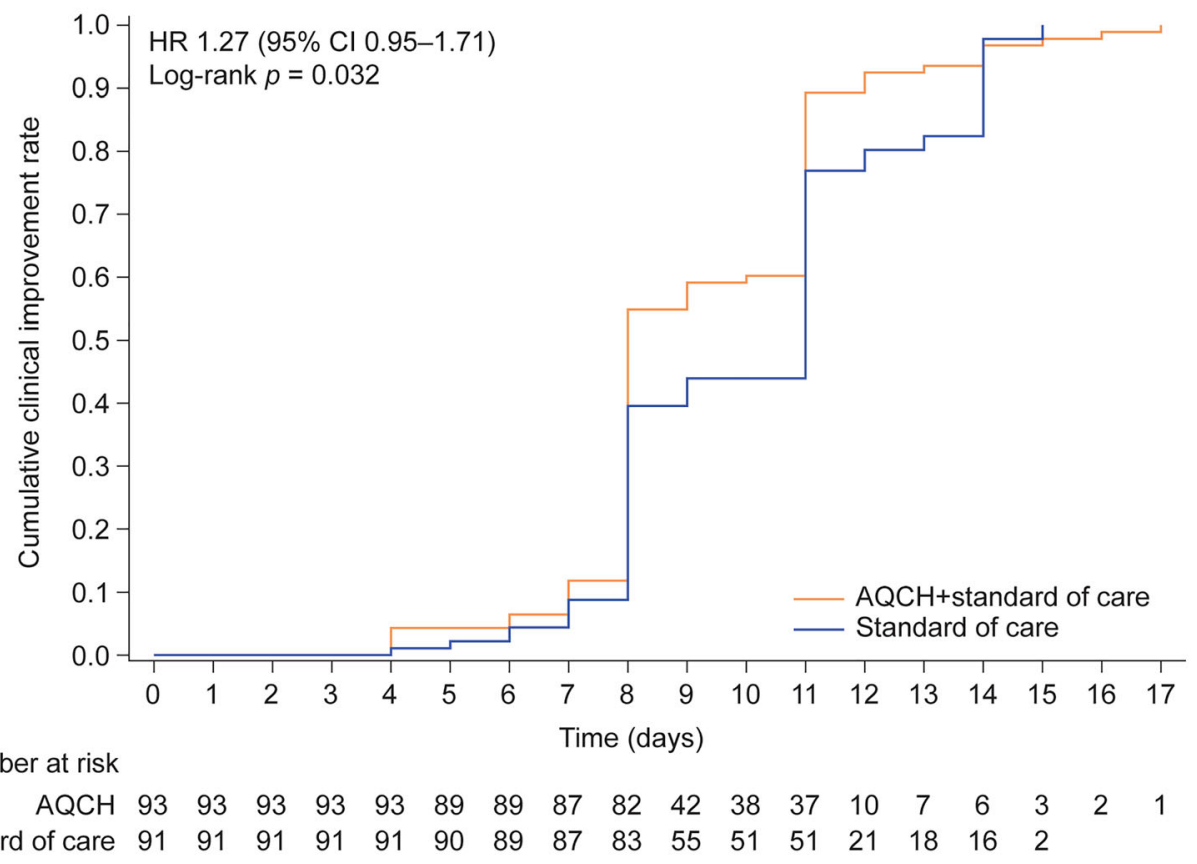

ii)

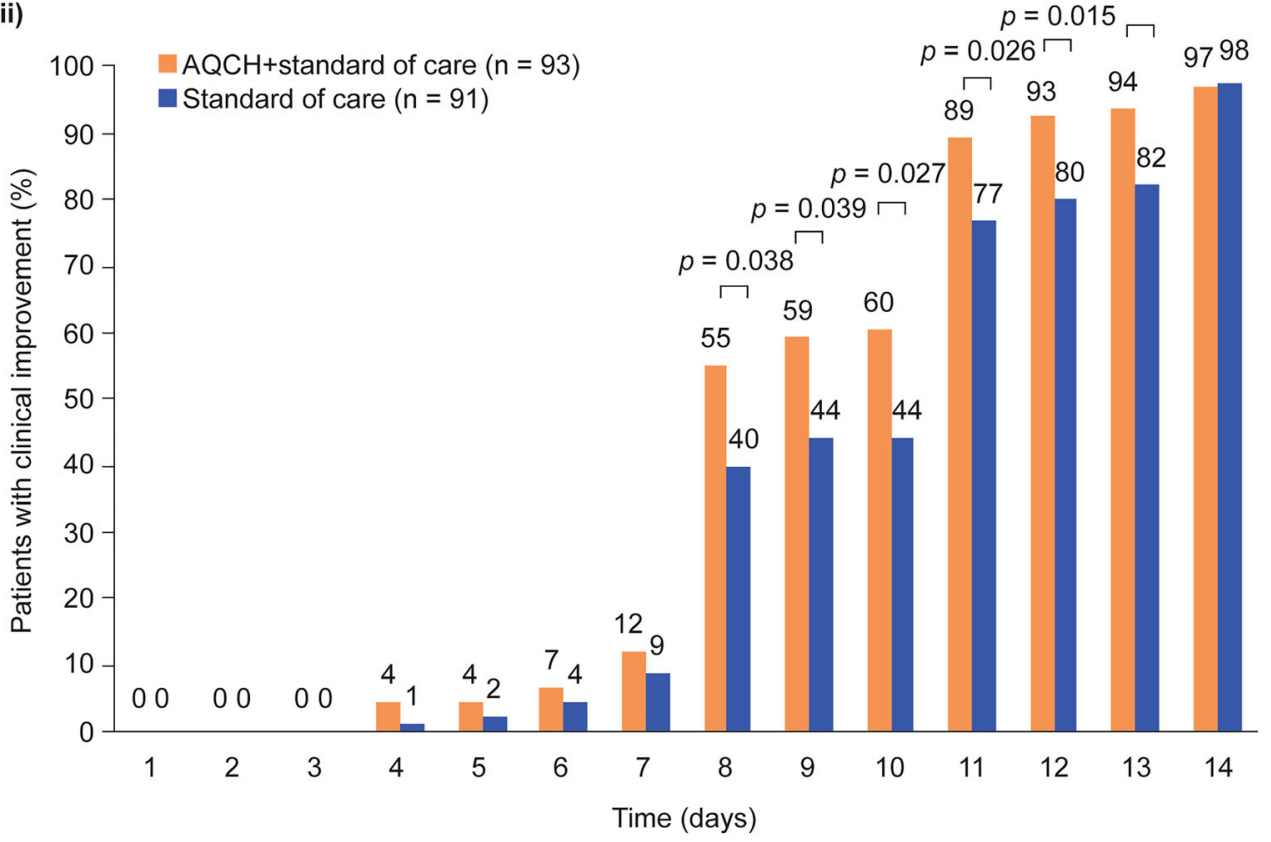

Fig. 2 Time to clinical improvement (i) and proportion of patients with clinical improvement (ii). Clinical improvement, as defined as meeting hospital discharge criteria or a 2-point improvement from time of enrollment in disease severity rating, in the per-protocol population. In ii, $p$ values are shown in cases of statistical significance (chisquared test/Fisher's exact test). $A Q C H$ aqueous extract of Cocculus birsutus 
i)
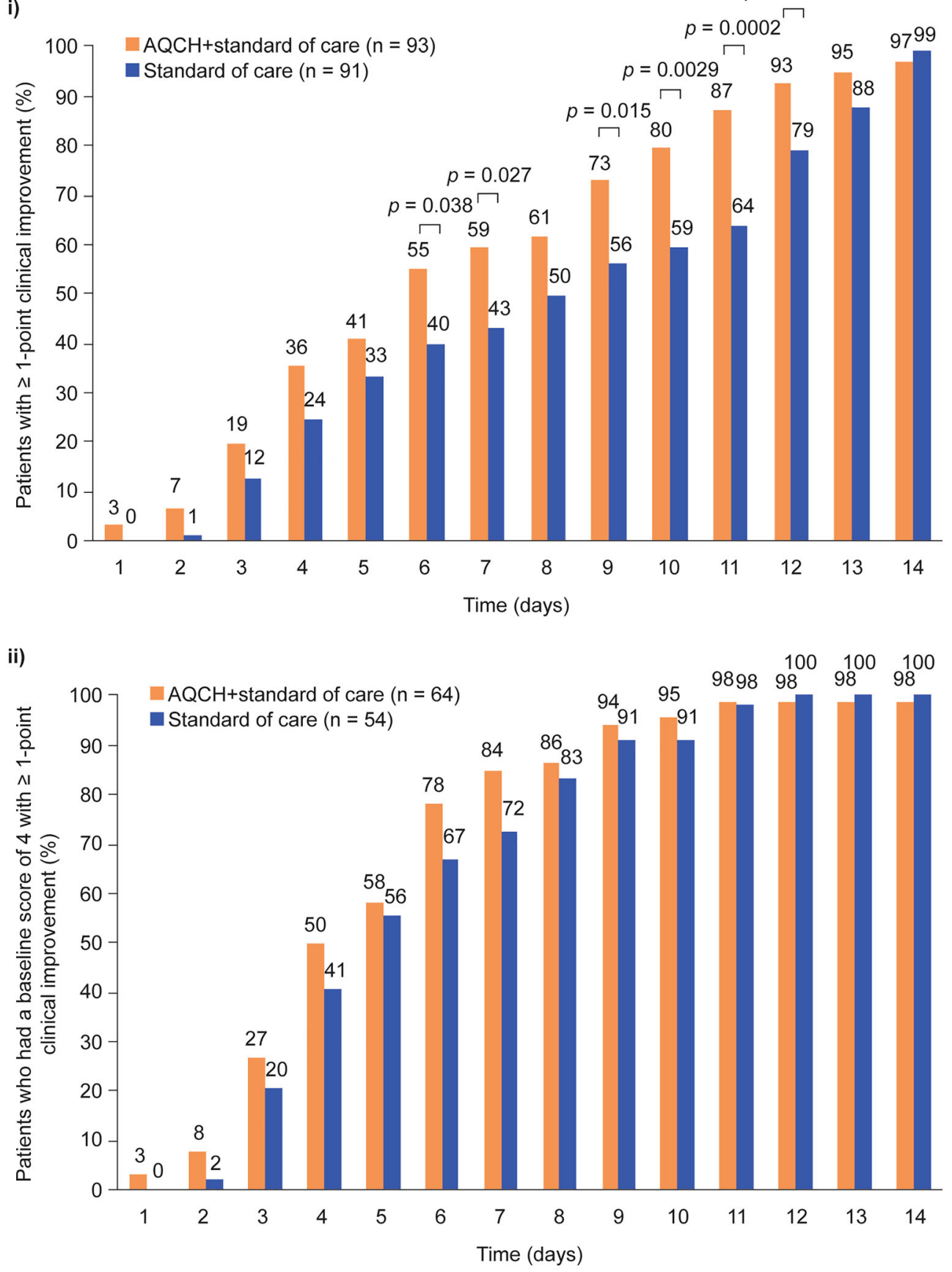

Fig. 3 Proportion of patients with at least a one-point clinical improvement in the per-protocol population (i) and in patients with a baseline score of 4 (ii). Clinical improvement was defined as $\geq 1$-point improvement from time of enrollment in disease severity rating. Patients with a score of 4 are hospitalized and require supplemental oxygen. $p$ values are shown in cases of statistical significance (chi-squared test/Fisher's exact test). $A Q C H$ aqueous extract of Cocculus hirsutus 


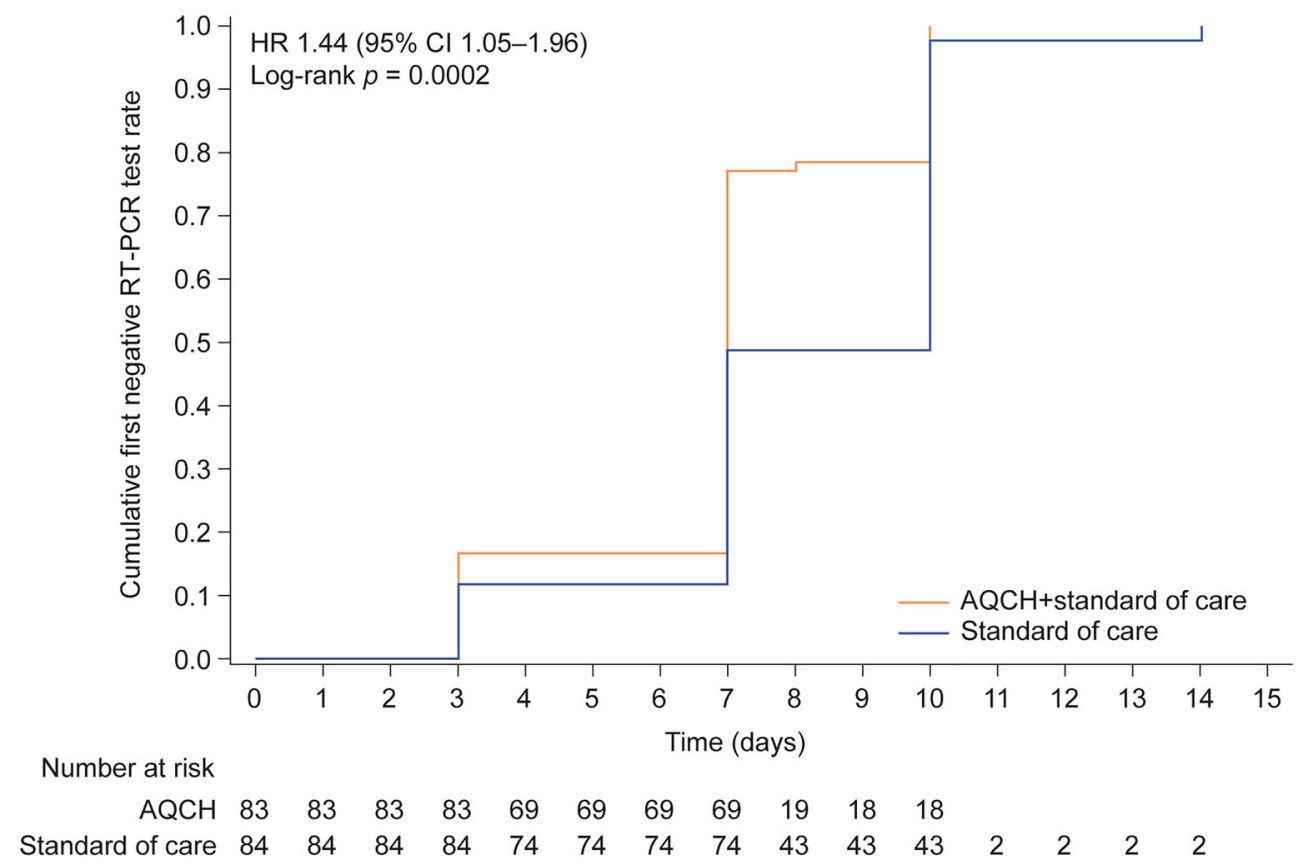

Fig. 4 Time to viral clearance. Time to first negative SARS-CoV-2 RT-PCR test in patients with no minor protocol deviations in RT-PCR testing (patients in whom

105 patients in the control group (Table 4). Most adverse events were mild in severity (15 of 17 events in the AQCH group versus 5 of 9 in the control group). Serious adverse events were reported in two patients, one in the $\mathrm{AQCH}$ group (two events: acute respiratory distress syndrome and liver injury) and one in the control group (acute respiratory distress syndrome), which resulted in the death of both patients. The event of liver injury was considered related to treatment, and study medication was permanently discontinued in this patient. No other adverse events were considered treatment related. No safety concerns were identified by the independent DSMB after review of the clinical data and laboratory findings.

\section{DISCUSSION}

This was a phase 2, open-label, randomized controlled trial to investigate the efficacy and safety of AQCH in hospitalized patients with moderate COVID-19. The primary endpoint of proportion of patients with clinical
RT-PCR was conducted on the protocol-scheduled days). $A Q C H$ aqueous extract of Cocculus hirsutus, SARS-CoV-2 severe acute respiratory syndrome coronavirus 2

improvement by day 14 was not significantly different with AQCH plus standard of care versus standard of care alone. However, treatment with AQCH plus standard of care resulted in significant benefits in time to clinical improvement, time to viral clearance, time to normalization of fever, and duration of hospitalization versus standard of care alone. AQCH tablets were well tolerated when used alongside standard of care in the management of patients with moderate COVID-19.

The primary endpoint for this study was selected based on World Health Organization (WHO) recommendations at the time of protocol preparation [20]. Patients in both treatment groups showed clinical improvement earlier than assumed during protocol development, which meant that by day 14 almost all the patients had recovered. Initial assumptions of treatment effect with standard of care were based on the limited experience of physicians treating COVID-19 at that time and reflect scarcity of published information about the natural course of the disease when the study 


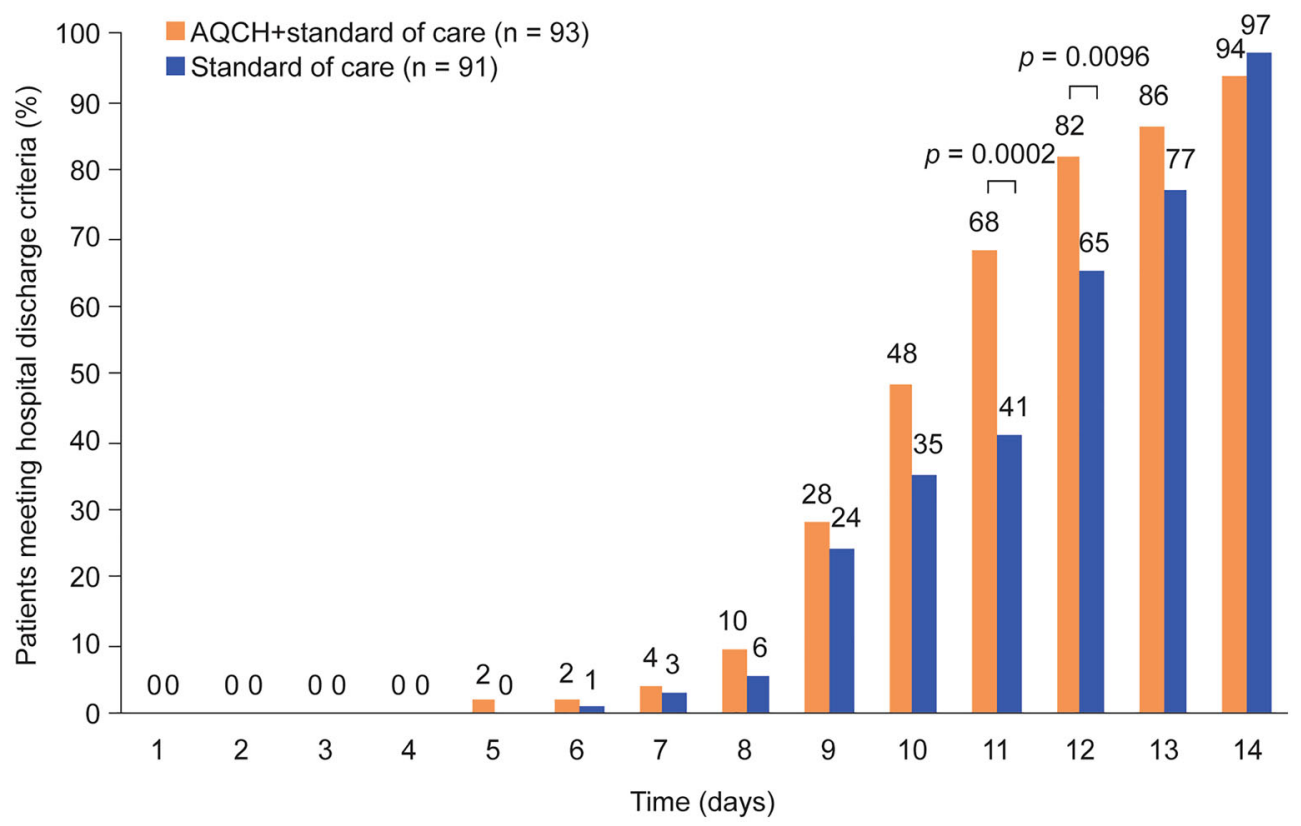

Fig. 5 Proportion of patients meeting hospital discharge criteria. Patients meeting hospital discharge criteria, as defined as resolution of symptoms and radiological improvement with documented viral clearance by RT-

was designed. This demonstrates the difficulties in designing clinical trials during the early stages of a pandemic and highlights the importance of time-to-event endpoints. In one study of remdesivir in hospitalized patients with predominantly severe COVID-19, the primary endpoint was changed after trial initiation from clinical status at day 15 to time to recovery [8]. The results of key secondary endpoints in our study, which investigated aspects of patient recovery over time, draw attention to the need to consider clinical trial results holistically and not through a narrow lens of primary endpoint alone.

Significant differences between the two treatment groups became apparent after the first week of treatment. In both groups, there was a substantial increase in the proportion of patients with clinical improvement on day 8 , reflecting the natural course of the disease and recovery of most patients during the second week of hospitalization. Patients who were receiving AQCH plus standard of care achieved clinical improvement on average 3 days earlier
PCR in two samples $\geq 24 \mathrm{~h}$ apart, in the per-protocol population. $p$ values are shown in cases of statistical significance (chi-squared test/Fisher's exact test). $\mathrm{AQCH}$ aqueous extract of Cocculus hirsutus

than those receiving standard of care alone and were eligible for discharge from hospital 1 day earlier. Of note, patients who were receiving supplemental oxygen at baseline appeared to reach a point at which they no longer required oxygen therapy faster in the AQCH group than in the control group. These findings have important implications for the care of hospitalized patients with COVID-19. In May 2021, India accounted for nearly half of the cases being reported worldwide, and hospitals in the country experienced a shortage of beds and oxygen supplies $[5,21]$. Earlier clinical improvement would lead to decreased healthcare resource utilization and faster hospital discharge, relieving some of the pressure on already stressed healthcare systems and increasing bed availability for new patients. Furthermore, in an acute setting, a reduction in recovery time could allow for individuals to return to work earlier, reducing the overall societal impact of the disease.

Improvement in clinical condition in the AQCH group was accompanied by significantly 
Table 4 Summary of treatment-emergent adverse events

\begin{tabular}{|c|c|c|}
\hline & $\begin{array}{l}\text { AQCH + standard of care } \\
(n=105)\end{array}$ & $\begin{array}{l}\text { Standard of care } \\
(n=105)\end{array}$ \\
\hline Any adverse event & $12(11)$ & $7(7)$ \\
\hline Adverse events leading to treatment discontinuation & $1(1)$ & 0 \\
\hline Adverse events related to treatment & $1(1)$ & 0 \\
\hline Adverse events leading to death & $1(1)$ & $1(1)$ \\
\hline Serious adverse events & $1(1)$ & $1(1)$ \\
\hline Acute respiratory distress syndrome & $1(1)$ & $1(1)$ \\
\hline Liver injury & $1(1)$ & 0 \\
\hline \multicolumn{3}{|l|}{$\begin{array}{l}\text { Adverse events by system organ class and preferred } \\
\text { term }\end{array}$} \\
\hline Gastrointestinal disorders & $1(1)$ & $1(1)$ \\
\hline Diarrhea & $1(1)$ & 0 \\
\hline Vomiting & $1(1)$ & $1(1)$ \\
\hline Hepatobiliary disorders & $1(1)$ & 0 \\
\hline Liver injury & $1(1)$ & 0 \\
\hline Investigations & $7(7)$ & $4(4)$ \\
\hline Increased alanine aminotransferase & $4(4)$ & $3(3)$ \\
\hline Increased aspartate aminotransferase & $2(2)$ & 0 \\
\hline Increased blood pressure & $2(2)$ & $1(1)$ \\
\hline Increased blood creatinine & $1(1)$ & 0 \\
\hline Increased transaminases & 0 & $1(1)$ \\
\hline Musculoskeletal and connective tissue disorders & $1(1)$ & 0 \\
\hline Pain in extremity & $1(1)$ & 0 \\
\hline Nervous system disorders & $3(3)$ & $1(1)$ \\
\hline Headache & $2(2)$ & $1(1)$ \\
\hline Dizziness & $1(1)$ & 0 \\
\hline Respiratory, thoracic, and mediastinal disorders & $1(1)$ & $2(2)$ \\
\hline Acute respiratory distress syndrome & $1(1)$ & $1(1)$ \\
\hline Dyspnea & 0 & $1(1)$ \\
\hline
\end{tabular}

Data are $n(\%)$

$A Q C H$ aqueous extract of Cocculus hirsutus 
earlier normalization of fever and a significantly shorter time to viral clearance, with patients receiving their first negative SARS-CoV-2 RTPCR test result on average 3 days earlier than patients in the control group. In a hospital setting, earlier viral clearance in patients with COVID-19 will decrease the number of infectious individuals and help to control spread of infection within the community.

Limited data are available for the efficacy of other antiviral drugs with emergency use authorization in India in patients with moderate COVID-19. In phase 3, open-label, clinical trials of remdesivir and favipiravir that included patients with moderate COVID-19, the primary endpoints were clinical status by day 11 (updated from proportion of patients discharged by day 14 in the original protocol) and time to cessation of oral shedding of SARS-CoV-2 virus, respectively $[11,16]$. Remdesivir treatment for 5 days was associated with significantly better clinical status at day 11 than standard of care alone in patients with moderate COVID-19; however, this effect was not seen in patients who received remdesivir for 10 days, and no significant benefit versus standard of care was found for time to clinical improvement or duration of hospitalization [11]. In patients with mild-to-moderate COVID-19, treatment with favipiravir was associated with a numerically shorter time to cessation of oral viral shedding versus standard of care, but this was not statistically significant [16].

The patients included in our study were relatively young in comparison with those in other clinical trials of antiviral drugs in moderate COVID-19 and had a relatively low frequency of comorbidities $(<20 \%)[11,16]$. In the international trial of remdesivir in patients with moderate COVID-19, over 50\% of patients had cardiovascular disease, and over $40 \%$ had hypertension or diabetes [11]. In the trial of favipiravir in Indian patients with mild-tomoderate COVID-19, 26\% of patients had a comorbid condition (diabetes, hypertension, and/or obesity), despite approximately twothirds of the patients having only mild disease [16]. The low rate of comorbidities in our study could be related to the exclusion of patients with concurrent medical conditions or uncontrolled, clinically significant systemic diseases in the protocol. It may also reflect a propensity of the investigators to recruit patients with less-severe disease to a clinical study of an investigational molecule.

Genotyping of SARS-CoV-2 strain was not performed as part of the study, which was conducted early in the pandemic before the appearance of the delta variant. No data are currently available on the effect of $\mathrm{AQCH}$ on different SARS-CoV-2 variants; however, it can be speculated that changes to the viral spike protein would be unlikely to influence the action of AQCH. In vitro studies indicate that AQCH acts after viral entry into the host cell, potentially by interfering with endosomal release of the viral RNA or its replication (manuscript in preparation).

Despite the practical challenges of conducting a clinical trial during a pandemic, the study recruited its target population of patients with moderate COVID-19 and demonstrated statistically significant differences between the two treatment groups in time to clinical improvement, viral clearance, fever resolution, and duration of hospitalization. The ordinal scale used for assessment of clinical status in the study is endorsed by the WHO, and has been used in other randomized controlled trials in patients with COVID-19 [2, 8, 10, 11]. Our study has a few limitations, which provide important lessons for designing future trials in patients with COVID-19. The evolving global understanding of the natural course of the disease during the first wave of the pandemic resulted in assumptions being made during selection of the primary endpoint that later became redundant. Interestingly, this has also been identified as a limitation in the DisCoVeRy study (a large randomized controlled trial of remdesivir in patients with severe COVID-19), in which the use of a fixed-time endpoint may have been responsible for the failure in primary outcome [22]. RT-PCR testing was not performed on each study day because, at that time, expert opinion was that daily testing should be avoided, given the limited availability of testing kits in India during the first wave of the pandemic. Had daily testing been carried out, we would have been able to identify the time point for viral 
clearance, and its relationship to clinical improvement, more precisely. Difficulties with RT-PCR testing were the cause of all major protocol deviations in the study, with tests not being conducted at the right time or patients with positive test results being discharged from hospital. Efficacy analyses were conducted in the per-protocol population, which excluded patients with major protocol deviations, to optimize clinical accuracy. A further limitation is that there were variations in the standard of care across different sites, with different hospitals following slightly different treatment protocols. There was limited use of steroids across the study population, possibly because data supporting reduced mortality with corticosteroid use in patients with COVID-19 had not yet been published when the study was initiated. Together, these limitations demonstrate the inherent difficulties of clinical trial design during a rapidly changing pandemic situation and the impact of an evolving understanding of disease pathophysiology and treatments on study outcomes. Future studies should investigate the effects of AQCH in patients with COVID-19 in larger trials, including in patients with milder disease, and should include radiological endpoints and assessment of oxygen utilization.

\section{CONCLUSIONS}

In conclusion, while no significant difference was seen in the primary endpoint of proportion of patients with clinical improvement by day 14 , the results presented here show that treatment with AQCH improves several clinical and viral parameters in hospitalized patients with moderate COVID-19. Oral treatment with AQCH tablets alongside standard of care was well tolerated, and resulted in significantly earlier normalization of fever, viral clearance, and clinical improvement, and significantly shorter duration of hospitalization, versus standard of care alone. Further investigation of the therapeutic potential of $\mathrm{AQCH}$ in patients with COVID-19 is warranted.

\section{ACKNOWLEDGEMENTS}

We thank the patients who volunteered for the study, and the teams at all participating centers. We are grateful to colleagues from ICGEB Italy, IIIM Jammu, DBT and Sun Pharma R\&D, whose tireless efforts helped bring this phytopharmaceutical drug to clinical development stage. We acknowledge Maulik Doshi (ex-Sun Pharmaceutical Industries Limited) for his support as a medical writer and medical monitor for the study, and Nilesh Kadam and Guruprasad Palekar (Sun Pharmaceutical Industries Limited) for overseeing study operations and site monitoring. Our thanks also to Dr N. Kumarasamy (Voluntary Health Services Hospital, Chennai, Tamil Nadu, India) for providing clinical expertise. Tata Consultancy Services (TCS) was responsible for clinical data management and biostatistics activities.

Funding. This work was supported by Sun Pharmaceutical Industries Limited, Mumbai, Maharashtra, India. Medical writing support for this manuscript was provided by Sarah Graham PhD of PharmaGenesis London, London, UK, with funding from Sun Pharmaceutical Industries Limited. The journal Rapid Service Fee was funded by Sun Pharmaceutical Industries Limited.

Authorship. All named authors meet the International Committee of Medical Journal Editors (ICMJE) criteria for authorship for this article, take responsibility for the integrity of the work as a whole, and have given their approval for this version to be published.

Authors' Contributions. Sadhna Joglekar and Shilpi Dhawan contributed to study conception and designed the study. Sadhna Joglekar, Suyog Mehta, and Shashank R. Joshi contributed to data analysis and interpretation. Shivakumar S. Iyer, Rohit Parate, Akash A. Khobragade, Rakesh Patil, Shashi Bhushan, Dnyanshwar M. Halnor, Girish C. Rajadhyaksha, and Kartikeya Parmar were the study investigators and contributed to data acquisition. All authors had full access to the data, 
critically reviewed the manuscript, and approved the final, submitted version.

Disclosures. Shivakumar S. Iyer, Rohit Parate, Akash A. Khobragade, Rakesh Patil, Shashi Bhushan, Dnyanshwar M. Halnor, Girish C. Rajadhyaksha, and Kartikeya Parmar declare that they have no conflict of interest. Sadhna Joglekar and Suyog Mehta are employees of Sun Pharmaceutical Industries Limited. Shilpi Dhawan was an employee of Sun Pharmaceutical Industries Limited at the time of the study and is currently an employee of Alvogen. Shashank R. Joshi has received advisory or speaker fees from Abbott, MSD, Novo Nordisk, Eli Lilly, Sanofi, USV, Boehringer Ingelheim, AstraZeneca, Novartis, Glenmark, Cipla, Zydus Cadila, Bayer, Franco-Indian Pharmaceuticals, Public Health Foundation of India, Sun Pharma, Twin Health, Biocon, DRL, Roche, Micro and Alkem.

Compliance with Ethics Guidelines. The study was conducted in accordance with the ethical principles of the Declaration of Helsinki, Good Clinical Practice guidelines issued by the Central Drugs Standard Control Organization, and the National Guidelines for Ethics Committees Reviewing Biomedical \& Health Research During COVID-19 Pandemic issued by the Indian Council of Medical Research (April 2020). Approval was obtained from the national regulatory agency and institutional ethics committee at each hospital before study initiation (B.J. Medical College and Civil Hospital ECR/72/Inst/GJ/2013/RR-19; Gandhi Medical College and Hamidia Hospital ECR/1055/Inst/ MP/2018; GGMC ECR/382/Inst/MH/2013/RR19; TNMC Nair Hospital ECR/22/Inst/Maha/ 2013/RR-19; BVDU Bharati Hospital and Research Centre ECR/313/Inst/MH/2013/RR-19; Vijay Vallabh Hospital Unit of Tirupati Life Care ECR/880/Inst/MH/2017/RR-20; Vijay Vallabh Hospital ECR/880/Inst/MH/2017; Bangalore Medical College \& Research Institute ECR/302/ Inst/KA/2013/RR-20). All participants provided written informed consent.

Data Availability. Data for this study are available from the corresponding author on reasonable request. Participant data will be provided after de-identification, and study documents will be redacted in compliance with applicable privacy laws.

Open Access. This article is licensed under a Creative Commons Attribution-NonCommercial 4.0 International License, which permits any non-commercial use, sharing, adaptation, distribution and reproduction in any medium or format, as long as you give appropriate credit to the original author(s) and the source, provide a link to the Creative Commons licence, and indicate if changes were made. The images or other third party material in this article are included in the article's Creative Commons licence, unless indicated otherwise in a credit line to the material. If material is not included in the article's Creative Commons licence and your intended use is not permitted by statutory regulation or exceeds the permitted use, you will need to obtain permission directly from the copyright holder. To view a copy of this licence, visit http://creativecommons.org/licenses/bync/4.0/.

\section{REFERENCES}

1. $\mathrm{Wu} \mathrm{Z}$, McGoogan JM. Characteristics of and important lessons from the coronavirus disease 2019 (COVID-19) outbreak in China: summary of a report of 72314 cases from the Chinese Center for Disease Control and Prevention. JAMA. 2020;323(13):1239-42. https://doi.org/10.1001/ jama.2020.2648.

2. Wang Y, Zhang D, Du G, et al. Remdesivir in adults with severe COVID-19: a randomised, double-blind, placebo-controlled, multicentre trial. Lancet. 2020;395(10236):1569-78. https://doi.org/10.1016/ S0140-6736(20)31022-9.

3. World Health Organization. WHO Coronavirus (COVID-19) Dashboard. https://covid19.who.int/ table. Accessed 27 Oct 2021.

4. Thiagarajan K. Why is India having a covid-19 surge? BMJ. 2021;373: n1124. https://doi.org/10. 1136/bmj.n1124.

5. Padma TV. Indian government should heed its scientists on COVID. Nature. 2021;593(7857):9. https://doi.org/10.1038/d41586-021-01140-6. 
6. Kifle ZD, Ayele AG, Enyew EF. Drug repurposing approach, potential drugs, and novel drug targets for COVID-19 treatment. J Environ Public Health. 2021;2021:6631721. https://doi.org/10.1155/2021/ 6631721.

7. Quek E, Tahir H, Kumar P, Hastings R, Jha R. Treatment of COVID-19: a review of current and prospective pharmacotherapies. Br J Hosp Med (Lond). 2021;82(3):1-9. https://doi.org/10.12968/ hmed.2021.0112.

8. Beigel JH, Tomashek KM, Dodd LE, et al. Remdesivir for the treatment of COVID-19-final report. N Engl J Med. 2020;383(19):1813-26. https://doi. org/10.1056/NEJMoa2007764.

9. WHO Solidarity Trial Consortium. Repurposed antiviral drugs for COVID-19-interim WHO Solidarity Trial results. N Engl J Med. 2020;384(6): 497-511. https://doi.org/10.1056/NEJMoa2023184.

10. McCreary EK, Angus DC. Efficacy of remdesivir in COVID-19. JAMA. 2020;324(11):1041-2. https:// doi.org/10.1001/jama.2020.16337.

11. Spinner CD, Gottlieb RL, Criner GJ, et al. Effect of remdesivir vs standard care on clinical status at 11 days in patients with moderate COVID-19: a randomized clinical trial. JAMA. 2020;324(11): 1048-57. https://doi.org/10.1001/jama.2020. 16349 .

12. Cai Q, Yang M, Liu D, et al. Experimental treatment with favipiravir for COVID-19: an open-label control study. Engineering (Beijing). 2020;6(10): 1192-8. https://doi.org/10.1016/j.eng.2020.03.007.

13. Chen C, Zhang Y, Huang J, et al. Favipiravir versus arbidol for COVID-19: a randomized clinical trial. MedRxiv. 2020. https://doi.org/10.1101/2020.03. 17.20037432.

14. Ivashchenko AA, Dmitriev KA, Vostokova NV, et al. AVIFAVIR for treatment of patients with moderate coronavirus disease 2019 (COVID-19): interim results of a phase II/III multicenter randomized clinical trial. Clin Infect Dis. 2020. https://doi.org/ 10.1093/cid/ciaa1176.

15. Goldman JD, Lye DCB, Hui DS, et al. Remdesivir for 5 or 10 days in patients with severe COVID-19. N Engl J Med. 2020;383(19):1827-37. https://doi. org/10.1056/NEJMoa2015301.
16. Udwadia ZF, Singh P, Barkate H, et al. Efficacy and safety of favipiravir, an oral RNA-dependent RNA polymerase inhibitor, in mild-to-moderate COVID19: a randomized, comparative, open-label, multicenter, phase 3 clinical trial. Int $\mathrm{J}$ Infect Dis. 2021;103:62-71. https://doi.org/10.1016/j.ijid. 2020.11 .142 .

17. González-Candelas F, Shaw M-A, Phan T, et al. One year into the pandemic: short-term evolution of SARS-CoV-2 and emergence of new lineages. Infect Genet Evol. 2021;92: 104869. https://doi.org/10. 1016/j.meegid.2021.104869.

18. Logesh R, Das N, Adhikari-Devkota A, Devkota HP. Cocculus hirsutus (L.) W.Theob. (Menispermaceae): a review on traditional uses, phytochemistry and pharmacological activities. Medicines (Basel). 2020;7(11):69. https://doi.org/10.3390/ medicines7110069.

19. Shukla R, Rajpoot RK, Poddar A, et al. Cocculus hirsutus-derived phytopharmaceutical drug has potent anti-dengue activity. Front Microbiol. 2021;12(3241): 746110. https://doi.org/10.3389/ fmicb.2021.746110.

20. WHO R\&D Blueprint Novel Coronavirus COVID-19 Therapeutic Trial Synopsis. February, 2020. https:// www.who.int/blueprint/priority-diseases/keyaction/COVID-19_Treatment_Trial_Design_ Master_Protocol_synopsis_Final_18022020.pdf. Accessed 22 Apr 2021.

21. World Health Organization. Weekly epidemiological update on COVID-19 - 4 May 2021. https:// www.who.int/publications/m/item/weeklyepidemiological-update-on-covid-19-4-may-2021. Accessed 10 May 2021.

22. Gyselinck I, Janssens W. Remdesivir, on the road to DisCoVeRy. Lancet Infect Dis. 2022;22(2):153-5. https://doi.org/10.1016/S1473-3099(21)00559-4.

\section{Publisher's Note}

Springer Nature remains neutral with regard to jurisdictional claims in published maps and institutional affiliations. 